\title{
Ressurgência: Um Estudo Estatístico de Temperatura e Salinidade da Boia $19^{\circ} 00^{\prime} \mathrm{s} 34^{\circ} 00^{\prime} \mathrm{w}$
}

\author{
Paulo Henrique Couto Simões'; José Fabiano da Serra Costa'; Marcello Montillo Provenzal; \\ Vinicius Layter Xavier'; Jorge Luiz de Jesus Goulart ${ }^{1}$
}

mprovenza@gmail.com

1. Universidade Estadual do Rio de Janeiro - UERJ, Maracanã, RJ.

Histórico do Artigo: 0 autor detém os direitos autorais deste artigo.

Recebido em: 13 de outubro de 2020 Aceito em: 01 de fevereiro de $2021 \quad$ Publicado em: 31 de agosto de 2021

Resumo: Os mais de sete mil quilômetros do litoral brasileiro constituem uma zona de riqueza ímpar do nosso país onde se concentra grande parte da população brasileira. É na região costeira onde se realiza grande parte da pesca, da navegação, da extração de petróleo, do lazer e do turismo do Brasil. Nesse cenário, um conhecimento das condições oceânicas existentes ao longo do nosso litoral torna-se cada vez mais necessário. Diversos programas incentivam e executam atividades relacionadas ao estudo dos oceanos. Nesse trabalho, será analisada a base de dados do GOOS-BRASIL que é um sistema nacional de observação dos oceanos. 0 objetivo do presente estudo é modelar as projeções das séries temporais entre 2005 e 2014 das temperaturas e salinidades médias a um metro de profundidade, coletadas da boia ATLAS (posição geográfica $19^{\circ} 00 \mathrm{~S} 34^{\circ} 00 \mathrm{~W}$ ) da rede PIRATA. Inicialmente foram feitas as análises de estacionariedade e sazonalidade, para posteriormente elaborar as previsões. Foram utilizados os modelos de alisamento exponencial e Box-Jenkins, os quais foram avaliados pelas métricas do Erro Quadrático Médio e da Média Absoluta Percentual de Erro. A temperatura teve o Alisamento Exponencial Linear de Brown e a salinidade os modelos AR (1) e Alisamento Exponencial Simples como melhores resultados.

Palavras-chave: Metoceanografia, Séries Temporais, Modelos de Alisamento, Modelos Box-Jenkins.

\section{Resurgence: A Statistical Study of Boove Temperature and Salinity $19^{\circ} 00^{\prime} \mathrm{s} 34^{\circ} 00^{\prime} \mathrm{w}$}

\begin{abstract}
The more than seven thousand kilometers of the Brazilian coast constitute an area of unparalleled wealth in our country, where a large part of the Brazilian population is concentrated. It is in the coastal region where a large part of fishing, navigation, oil extraction, leisure and tourism takes place in Brazil. In this scenario, a knowledge of the oceanic conditions existing along our coastline becomes increasingly necessary. Several programs encourage and carry out activities related to the study of the oceans. In this work, the GOOS-BRASIL database, which is a national ocean observation system, will be analyzed. The objective of the present study is to model the forecast of the time series between 2005 and 2014 of average temperatures and salinities at one meter depth, collected from the ATLAS buoy (geographical position $19^{\circ} 00 \mathrm{~S} 34^{\circ}$ 00W) of the PIRATA network. Initially, stationarity and seasonality analyzes were made, to later elaborate forecasts. The exponential smoothing and BoxJenkins models were used, which were evaluated by the metrics of the Mean Square Error and the Absolute Mean Percentage of Error. The temperature had Brown Linear Exponential Smoothing and salinity AR (l) and Simple Exponential Smoothing as the best results.
\end{abstract}

Keywords: Metoceanography, Times Series, Smoothing Models, Box-Jenkins Models. 


\section{Resurgencia: Estudio Estadístico de Temperatura y Salinidad Boove $19^{\circ} 00^{\prime} \mathrm{s} 34^{\circ} 00^{\prime} \mathrm{w}$}

Resumen: Los más de siete mil kilómetros de costa brasileña constituyen una zona de riqueza incomparable en nuestro país, donde se concentra gran parte de la población brasileña. Es en la región costera donde se desarrolla gran parte de la pesca, navegación, extracción de petróleo, ocio y turismo en Brasil. En este escenario, el conocimiento de las condiciones oceánicas existentes a lo largo de nuestra costa se vuelve cada vez más necesario. Varios programas fomentan y realizan actividades relacionadas con el estudio de los océanos. En este trabajo, se analizará la base de datos G00S-BRASIL, que es un sistema nacional de observación de los océanos. El objetivo del presente estudio es modelar la previsión de la serie temporal entre 2005 y 2014 de temperaturas y salinidades medias a un metro de profundidad, recogidas de la boya ATLAS (posición geográfica $19{ }^{\circ} 00 S 34{ }^{\circ} 00 \mathrm{~W}$ ) de la red PIRATA. Inicialmente se realizaron análisis de estacionariedad y estacionalidad, para luego elaborar pronósticos. Se utilizaron los modelos de suavizado exponencial y Box-Jenkins, los cuales fueron evaluados por las métricas del Error Cuadrático Medio y el Porcentaje de Error Medio Absoluto. La temperatura tuvo el Suavizado Exponencial Lineal Marrón y la salinidad AR (1) y el Suavizado Exponencial Simple como los mejores resultados.

Palabras clave: Metoceanografía, Series de Tiempo, Modelos de Enderezamiento, Modelos Box-Jenkins.

\section{INTRODUÇ̃̃o}

0 Brasil possui um litoral com mais de 7.400 km de extensão e, ao longo desta costa, são desenvolvidas diversas atividades de grande relevância socioeconômicas, tais como atividades portuárias, de pesca e aquicultura e de exploração de recursos minerais. Muitas das principais cidades brasileiras são litorâneas, tendo no turismo e nas atividades recreativas importantes fontes de renda, além de serem vulneráveis às variações do nível do mar para fins de saneamento, de defesa civil e demarcação do Patrimônio da União.

Conforme a Convenção das Nações Unidas sobre o Direito do Mar (ratificada por quase 100 países, inclusive o Brasil) todos os bens econômicos existentes no seio da massa líquida, sobre o leito do mar e no subsolo marinho, ao longo de uma faixa litorânea de 200 milhas marítimas de largura, na Zona Econômica Exclusiva (ZEE), constituem propriedade exclusiva do país ribeirinho. Em alguns casos, a Plataforma Continental (PC), prolongamento natural da massa terrestre de um Estado costeiro, ultrapassa essa distância, podendo estender a propriedade econômica do Estado a até 350 milhas marítimas. A ZEE mais a PC caracterizam a Amazônia Azul, medindo quase 4,5 milhões de $\mathrm{km}^{2}$, o que acrescenta ao país uma área equivalente a mais de $50 \%$ de sua extensão territorial.

Diversos programas, em nível mundial, incentivam e executam atividades relacionadas ao estudo dos oceanos. Um destes programas é o Global Sea Level Observing System (GLOSS, Sistema Global de Observação do Nível do Mar), coordenado pela organização World Meteorological Organization (WM0, Organização Meteorológica Mundial) e pela 
Intergovernmental Oceanographic Commission (IOC, Comissão Oceanográfica Intergovernamental). 0 GLOSS se destina a compor as atividades de estudar e monitorar os oceanos, e tem como objetivo primordial o estabelecimento de um sistema estratégico permanente para o fornecimento de informação de alta qualidade sobre o nível dos mares e a monitoração de suas mudanças globais.

0 equipamento básico de toda estação que monitora o nível do mar é o Marégrafo, que essencialmente consiste em um medidor que detecta e registra essa variável por meio de diversos sistemas, tais como flutuadores, sensores de pressão, contatos elétricos, pulsos acústicos, bolhas e radar. É extremamente importante que as estações realizem não só medições redundantes de nível do mar, como também medições de outros parâmetros meteorológicos e oceanográficos (salinidade, temperatura, umidade etc.).

Um requisito básico do GLOSS é que toda estação deve realizar e registrar medições com uma exatidão de centimetro a decimetro de centimetro sob todas as condições de maré, correntes de maré e todas as condições ambientais que possam ocorrer (chuva, temporais, ressacas e outras). 0 controle geodésico de cada estação deverá incluir a realização periódica de nivelamento geométrico de alta precisão, em intervalos de seis meses a um ano. As medições devem ser feitas seguindo as normas e especificações vigentes no Brasil (Decreto-Lei $n^{0}$ 243, 1967; Decreto-Lei $n^{\circ}$ 6.183, 1974). A boia localizada na posição geográfica $19^{\circ} 00^{\prime}$ S3400’W, objeto deste estudo, pertence à rede Pilot Research Moored Array in the Tropical Atlantic (PIRATA Pesquisa Piloto Ancorada no Atlântico Tropical). 0 projeto PIRATA, uma sub-rede do sistema GLOSS, é um programa de cooperação multinacional entre três países (Brasil, França e Estados Unidos), composto por boias fundeadas planejadas para monitorar uma série de variáveis dos processos de interação oceano-atmosfera no oceano Atlântico Tropical. As boias utilizadas são do tipo Autonomous Temperature Line Acquisition System (ATLAS, Sistema de Aquisição de Linha de Temperatura Autônoma).

0 propósito do deste trabalho é elaborar projeções das séries temporais no período compreendido entre janeiro de 2005 e dezembro de 2014 das temperaturas e salinidades médias a um metro de profundidade, coletadas da boia ATLAS da rede PIRATA. 0 uso das previsões permite que pesquisadores possam antever o fenômeno da ressurgência, acontecimento esse que tem maior consequência na situação socioeconômica local devido a pesca, além de conseguir compreender melhor o clima regional e suas variações. 


\section{Ressurgência}

0 principal fenômeno observado nesse estudo é 0 da ressurgência que consiste no afloramento de águas profundas com temperaturas frias, nas quais por estarem em uma região onde a luz solar não alcança (afótica) são ricas em nutrientes e sais minerais oriundos da deposição dos dejetos dos animais, seus corpos mortos, carapaças, etc., e pela ação das bactérias anaeróbicas tudo é remineralizado, sendo transformado nos chamados sais nutrientes, que através deste fenômeno subirão à superfície reiniciando o ciclo (Figura 1).

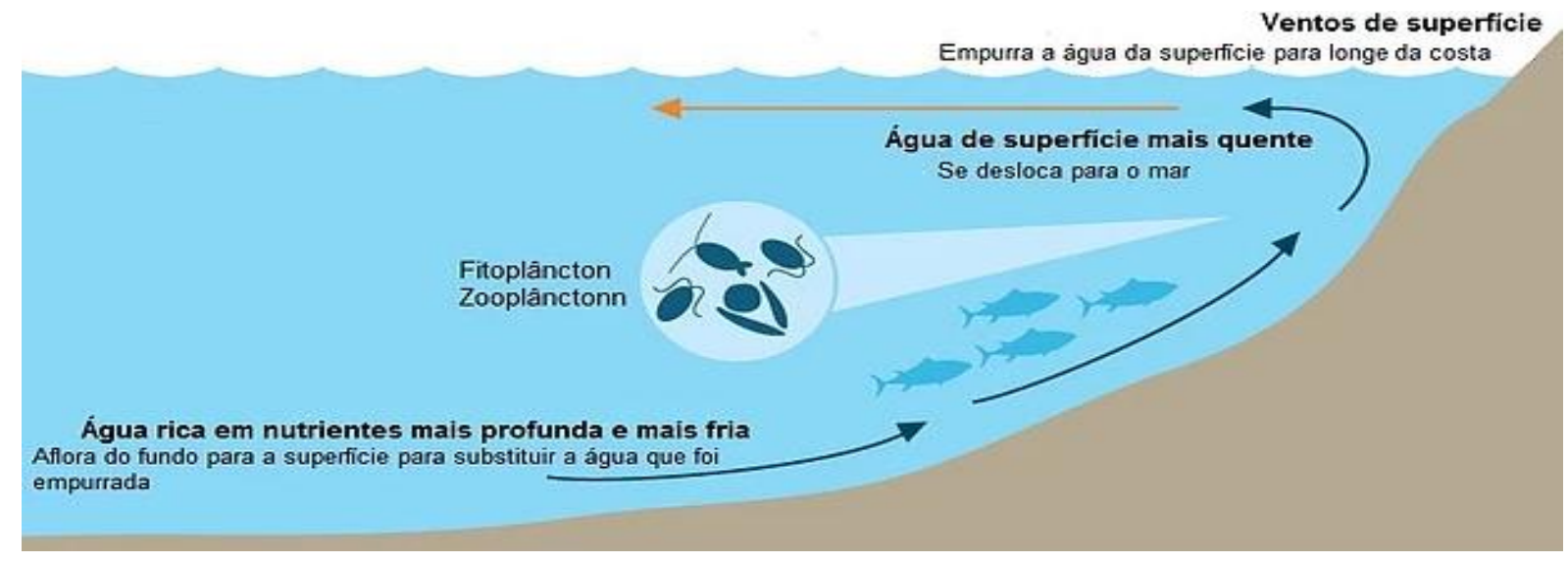

Figura 1. Fenômeno da ressurgência.

Fonte: Bate-papo com Netuno (2017). Imagem adaptada da Administração Nacional Oceânica e Atmosférica Departamento de Comércio dos Estados Unidos (National Oceanic and Atmospheric Administration U.S. Department of Commerce - NOAA).

Na superfície da água do mar vivem os organismos do plâncton, que flutuam nas correntes e são formados por algas microscópicas. 0s vegetais desta massa de organismos vivos são conhecidos como fitoplâncton, e os animais, como zooplâncton (estes seres dão início a cadeia alimentar). 0 volume de água empurrado para fora da costa é substituído por semelhante carregado do fundo para a superfície (ressurgência). Entre todos os fenômenos oceânicos, o de maior impacto sobre a situação socioeconômica é justamente a ressurgência. Nos mares tropicais a quantidade de fitoplâncton é pequena, o que justifica a pouca atividade pesqueira no Brasil. (MESQUITA, 2017).

\section{Sistema ATLAS}

Segundo Andrade e Costa (2011), atualmente no Brasil pode ser constatada uma crescente necessidade de obtenção de dados meteoceanográficos (meteoceanografia é a ciência que 
estuda os fluxos na atmosfera e sua interação com o oceano). Neste contexto, observa-se que essa demanda é mais expressiva nos setores de Óleo e Gás, Offshore, Naval, Portos e Estaleiros,

Mineração, Siderurgia, Usinas Hidrelétricas, Termelétricas e Nucleares; assim como, nos Institutos de Pesquisas Científicas, porque reduz a incerteza em especificações de engenharia, oferece ajuda no planejamento, permite a redução de custos e aumenta a segurança e a eficiência operacional. A obtenção de dados meteoceanográficos tem como objetivo aumentar a compreensão dos fenômenos e condições meteorológicas e oceanográficas, sendo que, tais dados são coletados principalmente em regiões onde possíveis projetos dos setores supracitados serão desenvolvidos, como por exemplo, plataformas de produção de petróleo, estaleiros, portos etc.

Além disso, com este conhecimento também é possível prevenir possíveis avarias a estruturas que venham ser instaladas em locais de interesse tanto em regiões costeiras ou offshore. Ao passo da necessidade de obtenção de parâmetros meteoceanográficos, ocorre também a necessidade de desenvolvimento de equipamentos nacionais para coleta de tais dados, já que, a maioria dos equipamentos disponíveis no mercado é de origem estrangeira, o que acarreta o aumento do custo e fragilidade do projeto ou, em alguns casos, pode até inviabilizar sua execução.

A rede operacional de observação do PIRATA coleta e armazena informações internamente em intervalos de 10 minutos (dados de alta resolução são recuperados durante a operação de manutenção anual das boias). As variáveis medidas são: vento de superfície, temperatura da superfície, salinidade, temperatura do ar, humidade relativa do ar, radiação de ondas curtas, precipitação, temperatura de sub-superfície, condutividade de sub-superfície e pressão a 300 e 500 m. A Figura 2 mostra um exemplo de boia ATLAS e a posição dela neste estudo.

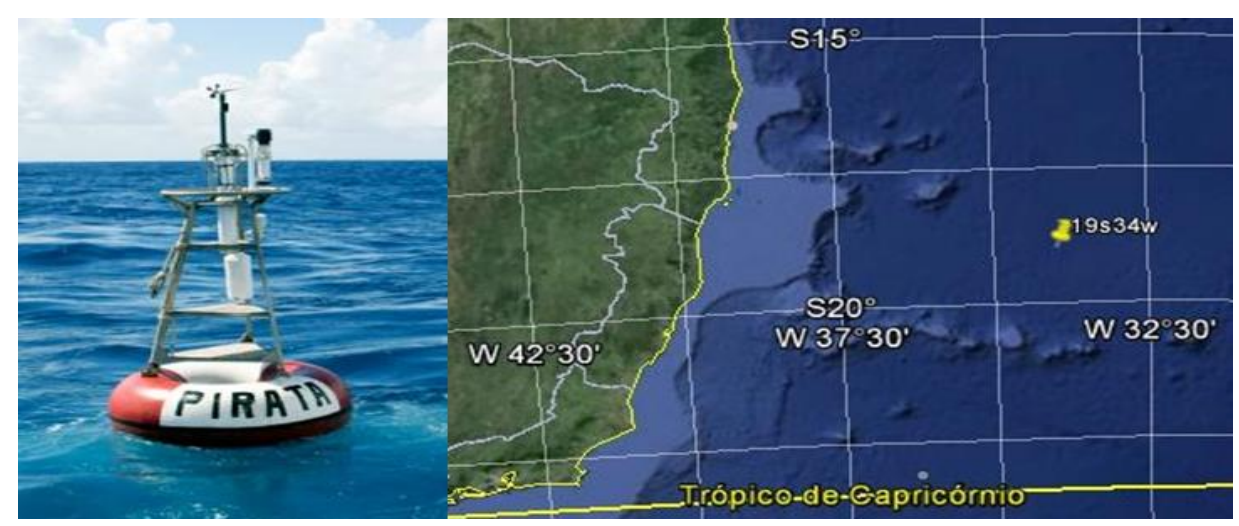

Revista Internacional de Ciências, v. 11, n. 02, p. 194-213, mai-ago, 2021 
Figura 2. Boia ATLAS e posição geográfica $\left(19^{\circ} 00^{\prime} \mathrm{S} 34^{\circ} 00^{\prime} \mathrm{W}\right)$.

Fontes: Marinha do Brasil (2019) e Google Earth (2015).

\section{Sistema ATLAS-B}

Tendo como base o projeto PIRATA, o Instituto 0ceanográfico da Universidade de São Paulo em apoio às atividades da componente oceânica do Instituto Nacional de Ciência e Tecnologia para mudanças climáticas, teve o interesse em uma boia fundeada para monitoramento meteoceanográfico da Bacia de Santos (ATLAS-B). 0 equipamento consiste em uma boia meteoceanográfica similar a usada no projeto PIRATA (BOURLÈS et al., 2008).

A estrutura da boia nacional denominada ATLAS-B possui forma toroidal com aproximadamente $2,3 \mathrm{~m}$ de diâmetro e foi confeccionada utilizando fibra de vidro e reforços internos de aço. A estrutura da boia do modelo MINITRIAXIS também foi confeccionada utilizando fibra de vidro, porém possui forma esférico-discoide e diâmetro de aproximadamente $1,2 \mathrm{~m}$.

Nas duas boias, existem aparatos que permitem a instalação de equipamentos para medição de parâmetros ambientais que não são medidos pela Unidade Móvel de Medição (UMM), tais como Perfilador de Corrente Doppler Acústico (ADCP), estação meteorológica, sensores de medição de temperatura e salinidade, entre outros. A UMM é composta por um sensor de medição de movimento; uma placa para aquisição e processamento dos dados do sensor de movimento, armazenamento e gerenciamento dos dados oriundos de outros sensores acoplados, um Sistema de Posicionamento Global (GPS) e uma fonte reguladora de tensão (Figura 3). Contudo, os componentes eletrônicos utilizados não são de origem nacional, e para redução dos custos foi realizada uma pesquisa de mercado que possibilitou a aquisição que atendessem aos objetivos específicos da construção do equipamento.

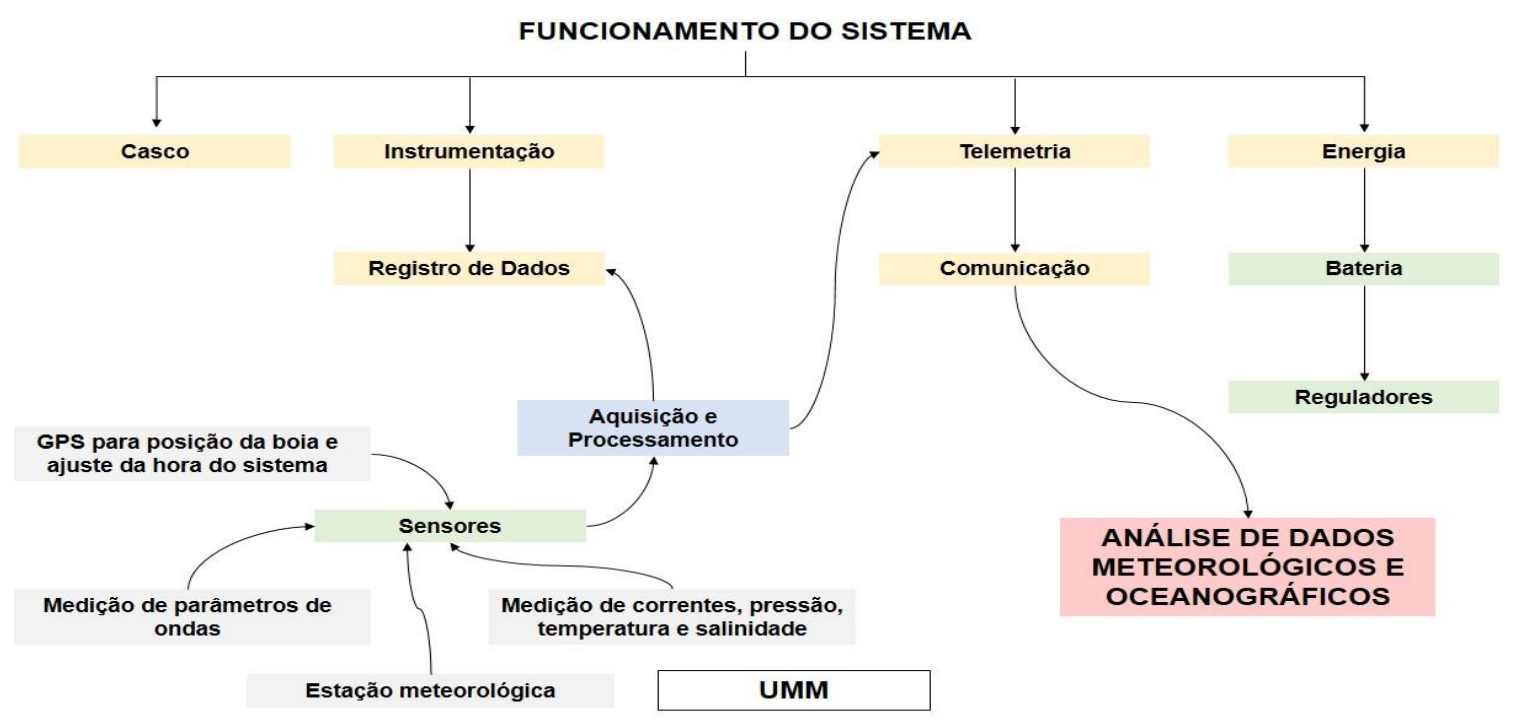


Figura 3. Representação esquemática do sistema ATLAS.

Fonte: Autores (2021).

\section{Estudos Quantitativos na 0ceanografia}

Um dos principais objetivos da oceanografia é conhecer a circulação oceânica. As medições por si só não fornecem todas as informações requeridas, pois elas depreendem um conjunto muito grande de fatores que influenciam as correntes, sendo primordial para analise o isolamento de cada um desses fatores. Em consequência, as medidas diretas de correntes em diversas profundidades no mar são normalmente acompanhadas de outras medições, de natureza oceanográfica e meteorológica. Os navios oceanográficos, plataformas, barcos e boias são os tradicionais suportes dos instrumentos e fonte primordial de informações para a construção de qualquer estudo.

Nos últimos 20 anos, pesquisadores vêm produzindo alguns trabalhos nessa área utilizando métodos quantitativos e, principalmente de análise de séries temporais, com estudos bastante acurados e, de eficácia comprovada.

Servain, Clauzet e Wainer (2005), do Instituto Oceanográfico da Universidade de São Paulo, tratam da análise multi-resolução por ondeletas no estudo de temperatura média mensal. Neste trabalho foram utilizados dados de alta resolução das boias PIRATA para avaliar as escalas de variabilidade temporal existentes na temperatura da superfície do mar. Os espectros de ondeletas são representados utilizando-se uma escala de cores (de azul claro a escuro) que indicam sua magnitude. A variabilidade temporal das séries foi analisada através da análise de ondeletas. A grande vantagem desta análise sobre a transformada de Fourier é a capacidade da ondeleta em alterar sua escala permitindo capturar componentes de diferentes frequências. As ondeletas conseguem descrever o comportamento local do sinal com maior precisão, enquanto a análise de Fourier, descreve o comportamento global do sinal. Isso faz com que o espectro de ondeletas seja menos afetado por uma não estacionariedade em um intervalo pequeno de tempo do que o espectro de Fourier.

No artigo de Molleri, Novo e Kampel (2009), os autores mostram que a técnica de análise de multi-resolução por ondeletas permitiu identificar as diferentes escalas de variação da série temporal da área da pluma do Rio Amazonas. Na análise multi-resolução por ondeletas, o sinal (série temporal) é decomposto por filtros passa-baixa e passa-alta em componentes referentes às baixas (aproximação) e às altas (detalhe) frequências, respectivamente. Os resultados desse estudo indicaram que a pluma apresenta uma forte variabilidade anual e sazonal. Esta sazonalidade está fortemente relacionada com o regime de vazão do Rio Amazonas e de seus 
afluentes. Os autores sugerem como forma de dar continuidade aos estudos da variabilidade espaço-temporal desta feição, aumentar o número de amostras da série temporal da área da pluma para períodos mais longos a fim de se identificar padrões de variabilidade em larga escala.

Paula e Souza (2010), do Laboratório de Meteorologia e 0ceanografia por Satélite do Centro Regional sul de Pesquisas Espaciais, analisam uma base de dados mensais da velocidade do vento na superfície e de sua componente zonal e meridional. A fim de obter uma estrutura mais detalhada da variabilidade do vento no tempo e frequência. Foi aplicada a Transformada de Ondas Contínuas (TOC) em séries temporais mensais. Para a identificação do padrão de variabilidade espaço-temporal, foi aplicada a Análises por Componentes Principais (ACP). A ACP é uma técnica bastante utilizada em análises de séries temporais, dada à possibilidade de demonstrar, em duas dimensões, a variação conjunta no tempo e espaço de um conjunto de dados. Este trabalho teve como principal objetivo analisar e descrever o comportamento temporal e espacial de dados de oceanografia e meteorologia, provenientes de satélites da região do 0ceano Atlântico Sudoeste através de métodos de análise de sinal.

Outra forma de análise de série temporal foi aplicada por Chechi e Oliveira Sanches (2013). Segundo o trabalho realizado, uma vez que a série de precipitação analisada apresentou pouca correlação nos seus dados dificultando o ajuste dos modelos SARIMA e Holt-Winters, optou-se por utilizar um modelo do tipo SARIMAX, o qual possibilita a inclusão de variáveis explicativas na forma de uma estrutura de regressão. Também, segundo os autores, a série sem nenhuma diferença não apresenta autocorrelação significativamente diferente de zero, assim como a série diferenciada de ordem um. Analisando os outros correlogramas, podemos identificar a utilização de um modelo sazonal, possivelmente com uma diferença de ordem 12 e com parâmetros negativos.

0 Quadro 1 apresenta outros trabalhos elaborados na área utilizando métodos quantitativos.

Quadro 1. Métodos quantitativos na área de climatologia oceanográfica.

\begin{tabular}{|c|c|c|}
\hline Tema & Técnicas/Métodos Utilizados & Autor(es) / Ano \\
\hline $\begin{array}{c}\text { Comparação de Séries } \\
\text { Temporais de Temperatura e } \\
\text { Salinidade da Água }\end{array}$ & $\begin{array}{c}\text { Séries Temporais; Testes de Quenouille, } \\
\text { Mélard e Roy, Coates e Diggle, e } \\
\text { Carmona e Wang }\end{array}$ & Echeverry e Toloi (2000) \\
\hline $\begin{array}{c}\text { Estimativa da Temperatura do } \\
\text { Ar }\end{array}$ & $\begin{array}{c}\text { Séries Temporais; Modelos de } \\
\text { Regressão e Métricas de Previsão }\end{array}$ & $\begin{array}{c}\text { Cavalcanti, Silva e Souza } \\
\text { (2006) }\end{array}$ \\
\hline $\begin{array}{c}\text { Meso-escala da Corrente da } \\
\text { Ressurgência }\end{array}$ & Modelo Regional Orientado por & Calado (2006) \\
\hline
\end{tabular}




\begin{tabular}{|c|c|c|}
\hline Climatologia da Ressurgência & $\begin{array}{c}\text { Análise Exploratória de Dados; Gráficos } \\
\text { da Temperatura da Superfície do Mar }\end{array}$ & $\begin{array}{c}\text { Silva, Dourado e Candella } \\
\text { (2006) }\end{array}$ \\
\hline $\begin{array}{c}\text { Temperatura da Superfície do } \\
\text { Oceano }\end{array}$ & $\begin{array}{c}\text { Modelo Oceânico R0MS; Análise } \\
\text { Exploratória de Dados (Gráficos e } \\
\text { Tabelas) }\end{array}$ & Cordeiro (2009) \\
\hline $\begin{array}{c}\text { Medições de Agitação } \\
\text { Marítima }\end{array}$ & $\begin{array}{c}\text { Modelo SWAN; Índice de Concordância, } \\
\text { Viés e EQM }\end{array}$ & Capitão e Fortes (2011) \\
\hline $\begin{array}{c}\text { Perda do Sinal Acústico na } \\
\text { Presença da Ressurgência }\end{array}$ & $\begin{array}{c}\text { Modelo Numérico Hidrodinâmico e } \\
\text { Modelo de Propagação Acústica }\end{array}$ & Codato et al. (2011) \\
\hline $\begin{array}{c}\text { Temperatura de Superfície do } \\
\text { Mar }\end{array}$ & $\begin{array}{c}\text { Séries Temporais; Modelos Box-Jenkins } \\
\text { e Redes Neurais Artificiais }\end{array}$ & Teixeira (2014) \\
\hline $\begin{array}{c}\text { Cálculo da Temperatura dos } \\
\text { 0ceanos }\end{array}$ & Série de Modos Normais & Laurindo e Polito (2015) \\
\hline $\begin{array}{c}\text { Variabilidade Superficial de } \\
\text { Temperatura e Altimetria }\end{array}$ & $\begin{array}{c}\text { Modelo Oceânico R0MS; Métricas de } \\
\text { Previsão e Destreza do Modelo }\end{array}$ & Mendonça et al. (2018) \\
\hline
\end{tabular}

Fonte: Autores (2019).

\section{Séries Temporais}

Uma série temporal é um conjunto de observações sobre uma variável obtidas sequencialmente ao longo do tempo e registradas em intervalos regulares. Deste modo, pode-se dizer que uma série temporal é a produção particular de um processo estocástico (BOX et al., 2015).

Para elaborar uma previsão temporal, deve-se tomar alguns passos. Primeiramente, avaliar a série graficamente, levantando alguns pressupostos. A seguir, aplicar testes para verificação de estacionariedade, sazonalidade e, $\log 0$ após, realizar o ajuste dos modelos (MORETTIN; TOLOI, 2006). Para avaliação dos métodos, utilizam-se as métricas de previsão (EQM e MAPE).

\section{Coeficiente de Spearman}

Os dados são analisados em pares que são postos ou que podem ser convertidos em postos, deste modo, o coeficiente de Separman mede a intensidade da relação entre variáveis ordinais (usando, em vez do valor observado, apenas a ordem das observações). Este teste pode ser usado para detectar algumas relações que não são lineares (CORDER; FOREMAN, 2014). A medida de correlação é dada por:

$$
\rho=1-\frac{6 \sum d^{2}}{n\left(n^{2}-1\right)}
$$

Se os postos da variável x são exatamente iguais aos postos da variável y, então todos as diferenças (d) serão zero, e $\rho$ será igual a 1. Os valores da série temporal (variável x) são colocados em ordem crescente e os postos correspondentes são examinados com base neste novo formato (variável y). Com o somatório das diferenças dos postos da variável x em relação a variável y, 
calcula-se o coeficiente. Se coeficiente $\rho$ de Spearman for menor que zero, a série pode ser descrita como estacionária.

\section{Teste Kruskal-Wallis}

Sazonalidade são oscilações de acréscimos ou decréscimos que se repetem em determinados períodos que podem coincidir com o calendário anual, mensal, semanal ou períodos específicos definidos. Resultam primariamente por naturalidade, mas também podem ser causadas pelo comportamento humano. Um estudo de sazonalidade tem por objetivo analisar o comportamento típico de uma série temporal. Para tanto, esta análise deve ser realizada em intervalos de tempos regulares.

0 processo de realização do Teste de Kruskal-Wallis tem início com a ordenação do conjunto de valores da variável, onde cada observação recebe um posto correspondente. Em seguida, somam-se os postos de cada grupo (no caso meses) e calcula-se a estatística H. Esta estatística pode ser aproximada por uma distribuição de probabilidade qui-quadrado, com (k 1) graus de liberdades, onde k é o número de períodos, nj é o número de casos em cada período j e N = $\sum$ nj (SIEGEL; CASTELLAN JR., 1988).

$$
\mathrm{H}=\frac{12}{\mathrm{n}(\mathrm{n}+1)} * \sum_{\mathrm{i}=1}^{\mathrm{k}} \frac{\mathrm{R}_{\mathrm{j}}^{2}}{\mathrm{n}_{\mathrm{j}}}-3(\mathrm{n}+1)
$$

Neste trabalho, os grupos (ou postos) foram os meses. Deste modo, todos os meses de janeiro formaram o grupo 1, fevereiro o grupo 2 e assim, sucessivamente, até dezembro que equivale ao grupo 12. Esta metodologia permite verificar se há pelo menos um mês fora do padrão da série. A hipótese nula, de não existência de sazonalidade é rejeitada se a estatística H for maior ou igual ao valor crítico retirado dado pela distribuição qui-quadrado.

\section{Modelos de alisamento}

Uma grande classe de métodos de previsão é a dos Métodos de Alisamento (Métodos de Suavização). Essas técnicas são usadas para se obter o padrão básico da série assumindo que os valores extremos são representados pela aleatoriedade (DOUKHAN, 2018). Deste modo, os modelos utilizados neste trabalho foram: Simples, Linear de Brown, Holt e Holt-Winters.

\section{Alisamento Exponencial Simples (AES)}

Os modelos AES são apropriados para séries que são caracterizadas localmente pelo seu nível e estacionárias. Consiste em ponderar todos os valores históricos com pesos 
Sucessivamente menores à medida que se afastam do valor mais recente (BROCKWELL; RICHARD, 2016). $\bar{Z}_{\mathrm{t}}$ é o valor alisado no estágio t e a previsão dos h valores futuros é dada pelo último valor exponencialmente suavizado. Dessa forma:

$$
\begin{aligned}
& \overline{\mathrm{Z}}_{\mathrm{t}}=\alpha \mathrm{Z}_{\mathrm{t}}+(1-\alpha) \mathrm{Z}_{\mathrm{t}-1} \\
& \hat{\mathrm{Z}}(\mathrm{h})=\overline{\mathrm{Z}}_{\mathrm{t}}
\end{aligned}
$$

\section{Alisamento Exponencial Linear de Brown (AELB)}

Não é apropriado o uso do AES quando uma série apresenta tendência linear, pois este modelo gera previsões que subestimam, ou superestimam, os valores reais. 0 AELB é semelhante ao AES, porém, indicado para séries que apresentam tendência. É usada uma única constante no processo de atualização do nível e da tendência, representados respectivamente pelos parâmetros $\hat{b}_{1, t}$ e $\hat{b}_{2, t}$. Consiste em calcular um segundo valor alisado, dado pela equação (BROCKWELL; RICHARD, 2016):

$$
\overline{\overline{\mathrm{Z}}}_{\mathrm{t}}=\alpha \overline{\mathrm{Z}}_{\mathrm{t}}+(1-\alpha) \overline{\overline{\mathrm{Z}}}_{\mathrm{t}-1}
$$

E os parâmetros das estimativas de nível e tendência são dados por:

$$
\begin{aligned}
& \hat{\mathrm{b}}_{1, \mathrm{t}}=2 \overline{\mathrm{Z}}_{\mathrm{t}}-\overline{\overline{\mathrm{Z}}}_{\mathrm{t}} \\
& \hat{\mathrm{b}}_{2, \mathrm{t}}=\frac{\alpha}{1-\alpha}\left(\overline{\mathrm{Z}}_{\mathrm{t}}-\overline{\overline{\mathrm{Z}}}_{\mathrm{t}-1}\right)
\end{aligned}
$$

Deste modo, a previsão final dos h valores futuros é dada por:

$$
\widehat{\mathrm{Z}}_{\mathrm{t}}(\mathrm{h})=\hat{\mathrm{b}}_{1, \mathrm{t}}+\hat{\mathrm{b}}_{2, \mathrm{t}}
$$

\section{Alisamento Exponencial de Holt (AEH)}

As características do AEH são similares a do AES, porém, além de suavizar o nível, uma nova constante é utilizada para a modelagem da tendência da série, resultando em dois coeficientes $\alpha$ e $\beta$ que representam, respectivamente, o nível e a tendência. A equação do modelo é representada por (BROCKWELL; RICHARD, 2016):

$$
\begin{aligned}
& \overline{\mathrm{Z}}_{\mathrm{t}}=\alpha \mathrm{Z}_{\mathrm{t}}+(1-\alpha)\left(\overline{\mathrm{Z}}_{\mathrm{t}-1}+\widehat{\mathrm{T}}_{\mathrm{t}-1}\right) \\
& \widehat{\mathrm{T}}_{\mathrm{t}}=\beta\left(\overline{\mathrm{Z}}_{\mathrm{t}}+\overline{\mathrm{Z}}_{\mathrm{t}-1}\right)+(1-\beta) \widehat{\mathrm{T}}_{\mathrm{t}-1}
\end{aligned}
$$

E a previsão final é dada por:

$$
\widehat{\mathrm{Z}}(\mathrm{h})=\overline{\mathrm{Z}}_{\mathrm{t}}+\mathrm{h} \widehat{\mathrm{T}}_{\mathrm{t}}
$$




\section{Alisamento Exponencial Sazonal de Holt-Winters (AESHW)}

É utilizada em séries temporais que apresentam padrões de comportamento mais complexos. Este modelo apresenta coeficientes para estimar o nível, a tendência e a sazonalidade, representados respectivamente por $\alpha, \beta$ e $\gamma$. A formulação do modelo sazonal aditivo é dada por (BROCKWELL; RICHARD, 2016):

$$
\begin{aligned}
& \widehat{\mathrm{S}}_{\mathrm{t}}=\gamma\left(\mathrm{Z}_{\mathrm{t}}-\overline{\mathrm{Z}}_{\mathrm{t}}\right)+(1-\gamma) \widehat{\mathrm{S}}_{\mathrm{t}-\mathrm{s}} \\
& \overline{\mathrm{Z}}_{\mathrm{t}}=\alpha\left(\mathrm{Z}_{\mathrm{t}}-\widehat{\mathrm{S}}_{\mathrm{t}-\mathrm{s}}\right)+(1-\alpha)\left(\overline{\mathrm{Z}}_{\mathrm{t}-1}+\widehat{\mathrm{T}}_{\mathrm{t}-1}\right) \\
& \widehat{\mathrm{T}}_{\mathrm{t}}=\beta\left(\overline{\mathrm{Z}}_{\mathrm{t}}+\overline{\mathrm{Z}}_{\mathrm{t}-1}\right)+(1-\beta) \widehat{\mathrm{T}}_{\mathrm{t}-1}
\end{aligned}
$$

E a previsão final é dada pela soma dos três parâmetros calculados:

$$
\widehat{\mathrm{Z}}(\mathrm{h})=\overline{\mathrm{Z}}_{\mathrm{t}}+\mathrm{h} \widehat{\mathrm{T}}_{\mathrm{t}}+\widehat{\mathrm{S}}_{\mathrm{t}+\mathrm{h}-\mathrm{s}}
$$

\section{Modelos Box-Jenkins}

0 modelo Autorregressivo Integrado de Médias Móveis (ARIMA) possui características flexíveis, permitindo ajustes e adaptações em seus parâmetros, contribuindo para que diferentes tipos de séries sejam representados, assumindo assim, uma estrutura de correlação linear entre os valores das séries temporais (B0X et al., 2015). A fórmula do modelo é dada por:

$$
\varphi(\mathrm{B})(1-\mathrm{B})^{d} \mathrm{Z}_{\mathrm{t}}=\theta(\mathrm{B}) \varepsilon_{\mathrm{t}}
$$

Onde $\varphi(\mathrm{B})$ é o polinômio autorregressivo, $\theta(\mathrm{B})$ é o polinômio de médias móveis, $\varepsilon_{\mathrm{t}}$ é um ruído branco com média zero e variância $\sigma^{2}$ e d é a ordem de integração (número de diferenças feitas à série para se tornar estacionária).

\section{Métricas de previsão}

Quando previsões periódicas são efetuadas, é importante monitorar os erros para determinar se estão dentro de limites razoáveis. Existem algumas métricas de previsão para avaliar a qualidade dos modelos propostos, das quais podem envolver valores absolutos ou percentuais.

0 EQM é muito útil na comparação de estimadores. Esta métrica é determinada somando os erros previstos ao quadrado e dividindo pelo número de previsões. Pode ser expresso pela equação (OLIVEIRA et al., 2010):

$$
\mathrm{EQM}=\frac{\sum\left(\mathrm{y}_{\mathrm{i}}-\widehat{\mathrm{y}}_{\mathrm{i}}\right)^{2}}{\mathrm{n}}
$$


Se a previsão do modelo é medida em milhares, os valores do EQM serão muito grandes. A utilização do MAPE é uma alternativa para resolução deste problema (é a média da diferença absoluta entre os valores previstos e atuais, expressa em percentagem). Assim, se existem previsões e valores reais para n períodos, o erro médio percentual absoluto é (OLIVEIRA et al., 2010):

$$
\mathrm{MAPE}=\frac{\sum\left|\frac{\mathrm{y}_{\mathrm{i}}-\hat{\mathrm{y}}_{\mathrm{i}}}{\mathrm{y}_{\mathrm{i}}}\right| * 100}{\mathrm{n}}
$$

\section{MATERIAIS E MÉTODOS}

As séries de salinidade e temperatura foram analisadas primeiramente de forma visual através de suas linhas temporais. Em seguida, foi aplicado o coeficiente de Spearman para verificação de tendência e o teste de kruskal-Wallis para investigação da sazonalidade. As projeções tiveram como base os métodos de alisamento exponencial e Box-Jenkins. Para averiguação dos melhores resultados, o EQM e o MAPE foram utilizados. Os dados foram extraídos do Banco de Dados 0ceanográficos (2015), que faz parte do sistema GLOSS.

Para elaboração deste trabalho, foram utilizados os softwares Microsoft Excel e $\mathrm{R}$ Project, pacotes forecast e stats (R CORE TEAM, 2019). Todas as previsões foram feitas um período à frente e realizadas por professores e pesquisadores da Universidade do Estado do Rio de Janeiro (UERJ), cidade do Rio de Janeiro, RJ.

\section{RESULTADOS E DISCUSSÃ0}

Com o intuito de observar as características das variações das séries temporais, como por exemplo, sazonalidade, estacionariedade, variabilidade e pontos atípicos, é de extrema importância a construção de um gráfico. 0 comportamento das séries, ao longo do período observado, será analisado inicialmente através da análise descritiva dos dados. Nota-se que as séries não apresentam tendência de crescimento ou decrescimento, indicando serem séries estacionárias, conforme o Gráfico 1. 
Gráfico 1. Valores Observados para Temperatura e Salinidade Mensal.

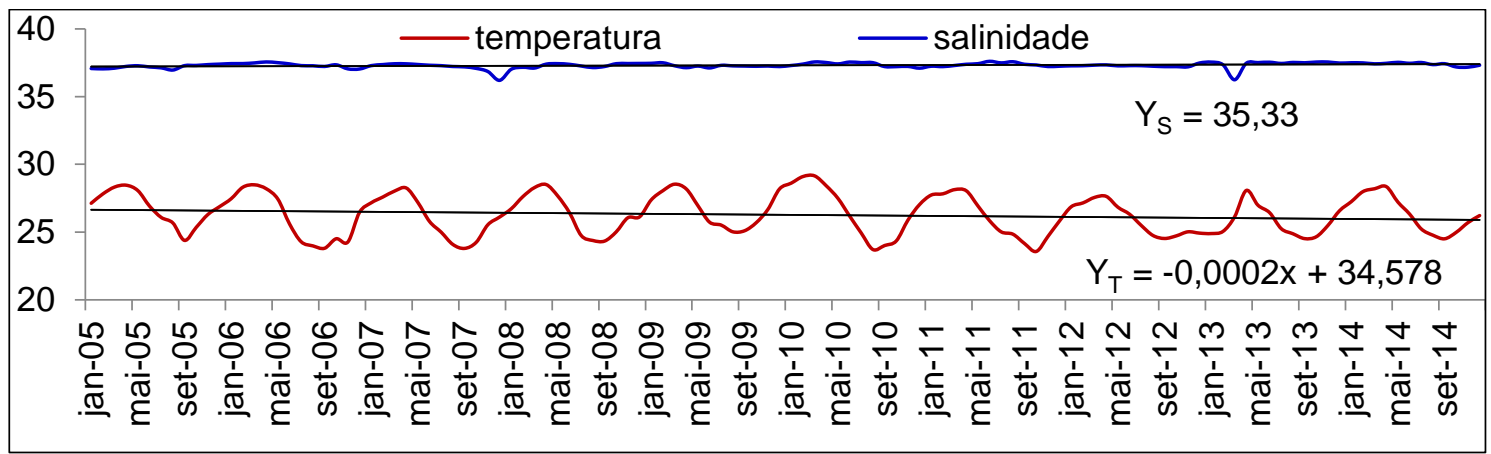

Fonte: Programa Global 0cean Observing System (2015).

Na linha da salinidade $\left(\mathrm{Y}_{\mathrm{S}}\right)$, há períodos que chamam a atenção por terem valores relativamente diferentes do resto da série. Inicialmente, não há explicação para isso, e pode ser um erro de medição. Observa-se também as retas de regressão que compõem o Gráfico 1 salinidade $\left(\mathrm{Y}_{\mathrm{S}}\right)$ e temperatura $\left(\mathrm{Y}_{\mathrm{T}}\right)$ - não possuem indicação de crescimento ou decrescimento, visto que seus coeficientes angulares são aproximadamente nulos.

Realizou-se uma análise de componente de tendência para verificar se as variáveis temperatura e salinidade apontam a não existência dela nas séries em estudo. Nesta análise, utilizou-se o coeficiente de correlação de Spearman, que para valores negativos indica que não existe tendência, confirmando-se que as séries temporais em estudo não apresentam movimentos fortes de crescimento ou decrescimento. 0 Quadro 2 apresenta os resultados.

Quadro 2. Coeficiente de Correlação de Spearman.

\begin{tabular}{|c|c|c|}
\hline Variável & Valor de $\rho$ & Conclusão \\
\hline Temperatura & $-0,142$ & Estacionária \\
\hline Salinidade & $-0,017$ & Estacionária \\
\hline
\end{tabular}

Fonte: Autores (2020).

Observou-se que a salinidade nessa área do oceano atlântico não possui uma variação cíclica que é característica das séries que possuem sazonalidade. É possível observar que tanto visualmente, quanto que por meio do teste de Kruskal-Wallis, não foi captada nenhuma componente sazonal (Quadro 3). Já na estatística teste para a temperatura média mensal é rejeitada a hipótese nula de que, em média, os doze meses sejam iguais. Dessa forma, fica evidente que pelo menos um mês possui uma temperatura média diferente das demais. $0 \mathrm{~s}$ resultados compilados para a temperatura e salinidade estão descritos a no Quadro 3.

Quadro 3. Teste de Kruskal-Wallis. 


\begin{tabular}{|c|c|c|c|c|c|c|}
\hline Variável & $\begin{array}{c}\text { Valor } \\
\text { Calculado }\end{array}$ & $\begin{array}{c}\text { Valor } \\
\text { Tabelado }\end{array}$ & p-valor & $\begin{array}{c}\text { Graus de } \\
\text { Liberdade }\end{array}$ & $\begin{array}{c}\text { Nível de } \\
\text { Significância }\end{array}$ & Conclusão \\
\hline Temperatura & 97,246 & 19,675 & $6,3 \times 10^{-16}$ & 11 & 0,05 & Sazonal \\
\hline Salinidade & 11,691 & 19,675 & 0,387 & 11 & 0,05 & $\begin{array}{c}\text { Não } \\
\text { Sazonal }\end{array}$ \\
\hline
\end{tabular}

Fonte: Autores (2020).

As séries de temperatura e salinidade médias mensais foram divididas em dois períodos. 0 período entre janeiro de 2005 e dezembro de 2012 foi utilizado como base de treino para a estimação dos parâmetros dos modelos, enquanto, o período entre janeiro de 2013 e dezembro de 2014 foi utilizado como base de teste para a verificação. 0s parâmetros da base de treino foram calculados com base nos menores valores resultantes/oriundos do EQM e MAPE, e usados na base de teste. Contudo, para os modelos AESHW e ARIMA, usou-se o Software R (JONATHAN; KUNK-SIK, 2008) para se obter os valores. 0s Quadros 4 e 5 apresentam os resultados.

Quadro 4. Valores das Constantes de Alisamento.

\begin{tabular}{|c|c|c|c|c|c|c|}
\hline \multirow{3}{*}{ Modelos } & \multicolumn{6}{|c|}{ Constantes } \\
\hline & \multicolumn{3}{|c|}{ Temperatura } & \multicolumn{3}{|c|}{ Salinidade } \\
\hline & $\alpha$ & $\beta$ & $\gamma$ & $\alpha$ & $\beta$ & $\gamma$ \\
\hline AES (EQM) & 0,99 & - & - & 0,82 & - & - \\
\hline AES (MAPE) & 0,99 & - & - & 0,99 & - & - \\
\hline AELB (EQM) & 0,95 & - & - & 0,43 & - & - \\
\hline AELB (MAPE) & 0,99 & - & - & 0,99 & - & - \\
\hline AEH (EQM) & 0,90 & 0,90 & - & 0,80 & 0,10 & - \\
\hline AEH (MAPE) & 0,90 & 0,90 & - & 0,90 & 0,10 & - \\
\hline AESHW & 0,78 & 0,0007 & 0,98 & 0,70 & 0,00 & 0,62 \\
\hline
\end{tabular}

Fonte: Autores (2020).

Quadro 5. Valores dos Parâmetros do ARIMA.

\begin{tabular}{|c|c|c|c|}
\hline Variável & p & d & q \\
\hline Temperatura & 2 & 0 & 2 \\
\hline Salinidade & 1 & 0 & 0 \\
\hline
\end{tabular}

Fonte: Autores (2020).

0 modelo ARIMA para temperatura pode ser definido como ARMA(2,2), autorregressivo e de média móvel de ordens $p=2$ e q = 2, e a salinidade como AR(l) - autorregressivo de ordem $p$ 
= 1. Como as duas variáveis são estacionárias, não foi necessária nenhuma diferenciação nas séries temporais (ou seja, $\mathrm{d}=0$ ).

Os Gráficos 2 e 3 apresentam a comparação entre os valores observados e as previsões obtidas pelos respectivos parâmetros EQM e MAPE em cada modelo aplicado para variável temperatura dentro do período compreendido entre janeiro de 2013 e dezembro de 2014.

Gráfico 2. Previsão da Temperatura pelos Parâmetros Avaliados no EQM.

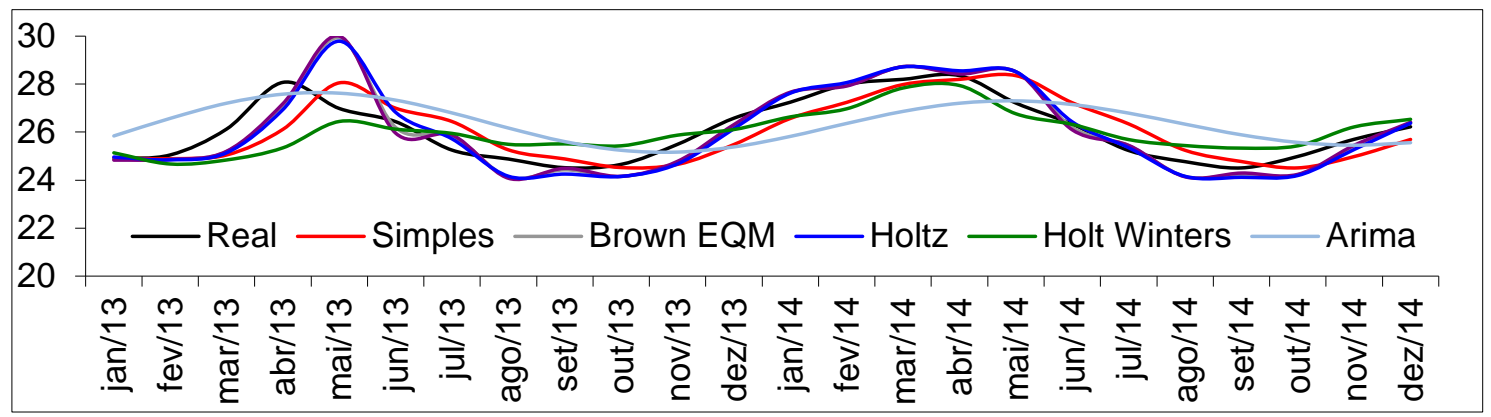

Fonte: Autores (2020).

Gráfico 3. Previsão da Temperatura pelos Parâmetros Avaliados no MAPE.

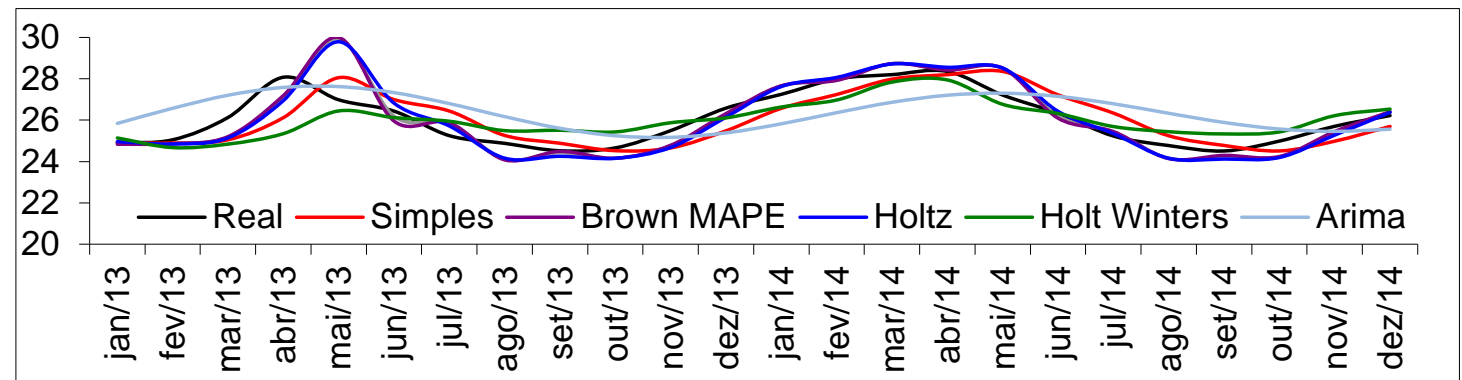

Fonte: Autores (2020).

0s Gráficos 4 e 5 apresentam a comparação entre os valores observados e as previsões obtidas pelos respectivos parâmetros EQM e MAPE em cada modelo aplicado para variável salinidade dentro do período compreendido entre janeiro de 2013 e dezembro de 2014.

Gráfico 4. Previsão da Salinidade pelos Parâmetros Avaliados no EQM.

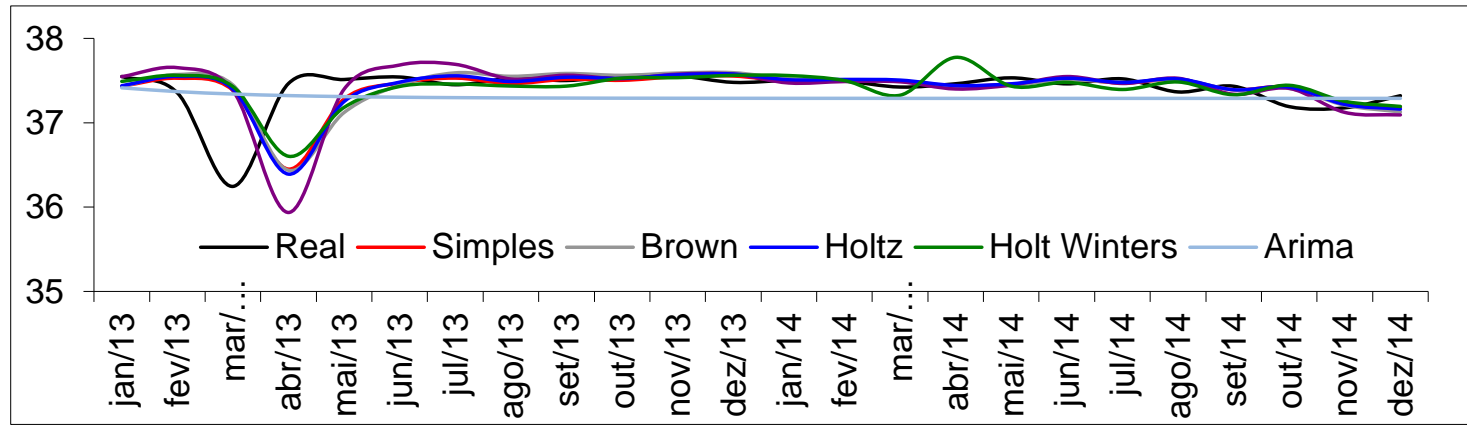

Fonte: Autores (2020). 
Gráfico 5. Previsão da Salinidade pelos Parâmetros Avaliados no MAPE.

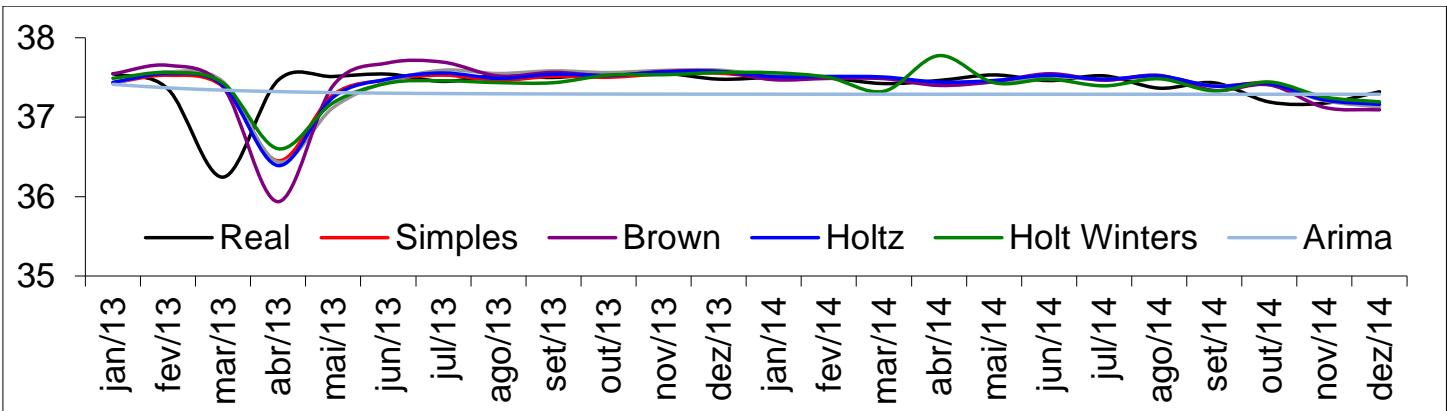

Fonte: Autores (2020).

De acordo com LEWIS (1997), o potencial de previsão do MAPE pode ser relacionado da seguinte forma: menor que $10 \%$ são previsões potencialmente muito boas; entre $10 \%$ e $20 \%$ são previsões potencialmente boas; entre $20 \%$ e $30 \%$ são previsões potencialmente razoáveis; e maior que 30\% são previsões potencialmente imprecisas.

Na aplicação dos dados aos métodos, por intermédio do EQM e do MAPE, identificou-se que o modelo de AELB é o mais indicado para representar o comportamento da série temporal das temperaturas médias mensais, por esta apresentar forte componente sazonal e não apresentar tendência. Para salinidade, os modelos ARIMA e AES são os mais indicados para a série temporal, pois estes não consideram o componente sazonal e a tendência. Tais resultados podem ser observados através do Quadro 5 .

Quadro 5. Valores das Métricas de Previsão

\begin{tabular}{|c|c|c|c|c|}
\hline \multirow{2}{*}{ Modelos } & \multicolumn{2}{|c|}{ Temperatura } & \multicolumn{2}{c|}{ Salinidade } \\
\cline { 2 - 5 } & EQM & MAPE & EQM & MAPE \\
\hline AES & 0,6599 & 2,5758 & 0,1069 & 0,4442 \\
\hline AELB & 0,6577 & 2,1181 & 0,1207 & 0,5415 \\
\hline AEH & 0,6580 & 2,1988 & 0,1150 & 0,4617 \\
\hline AESHW & 0,6802 & 4,2161 & 0,1075 & 4,2161 \\
\hline ARIMA & 1,2107 & 3,8610 & 0,0816 & 0,5568 \\
\hline
\end{tabular}

Fonte: Autores (2020).

\section{CONSIDERAÇÕES FINAIS}

Cerca de 25\% das capturas globais de peixes são oriundas de cinco locais que abrangem apenas 5\% da área oceânica total. As ressurgências que são conduzidas por correntes costeiras, ou divergentes nos oceanos abertos e têm grande efeito sobre as águas enriquecendo-as em 
nutrientes e melhorando os rendimentos da pesca. Entre todos os fenômenos oceânicos, este é o de maior influência sobre a situação socioeconômica das regiões pesqueiras (MESQUITA, 2017).

No Brasil a ressurgência é mais forte em Cabo Frio (mas acontece com menos força do Rio de Janeiro até Vitória, Espírito Santo), em função da topografia (mudança da linha da costa que vai no sentido Norte-Sul para Leste-0este) e pela profundidade da plataforma na região (MESQUITA, 2017). Deste modo, elaborar projeções para variáveis da ressurgência como temperatura e salinidade contribuem no movimento pesqueiro econômico das cidades, ajuda a entender melhor o clima da região sudeste e suas mudanças climáticas.

0 método de Alisamento Exponencial é uma técnica de previsão, que considera o princípio da estimação recursiva, e não exige qualquer transformação nos dados originais para que, por exemplo, respeitem uma distribuição normal ou sejam estacionários. A aplicação deste método concentra-se apenas no uso de fórmulas com as quais as previsões são obtidas através de alisamento dos valores passados. Assim sendo, as principais características deste método estão relacionadas a facilidade de uso e a aplicabilidade a um grande número de séries temporais. A utilização desse tipo de metodologia pode render resultados muito satisfatórios dependendo de condições como tendência, sazonalidade, ciclo e componente aleatório, além de permitir uma boa compreensão dos resultados.

A modelagem Box-Jenkins é comumente aplicada em processos lineares não estacionários. Quando as séries temporais não são estacionárias, ou seja, há movimentos de tendência seja de crescimento ou decaimento de qualquer natureza, para torná-las estacionárias, deve-se aplicar um número determinado de diferenças entre seus dados de ordem d, geralmente uma ou duas vezes. Para este processo o modelo é representado por ARIMA (p, d, q). 0 número de vezes que a série original tem que ser diferenciada antes de resultar em uma série estacionária é denominada ordem de integração.

Em geral, para a previsão de variáveis meteorológicas, utilizam-se modelos numéricos da circulação geral da atmosfera e oceano. Estes modelos necessitam de uma grande quantidade de informações e capacidade de processamento computacional. Como resultados são produzidas previsões de temperatura, vento, umidade e outros, em uma escala de tempo mais curta. Podese considerar que nenhum modelo obteve fraco desempenho (LEWIS, 1997). Para a temperatura, o modelo que obteve melhor ajuste foi o Alisamento Exponencial Linear de Brown, e para salinidade, os modelos AR (1) e Alisamento Exponencial Simples foram os que apresentaram melhores resultados.

A sugestão para prosseguimento deste trabalho, ainda no âmbito da previsão de séries temporais seria a utilização das técnicas de Machine Learning (JAMES, et al., 2013). Também 
seria interessante testar outras boias com diferentes localizações geográficas e verificar possíveis correlações entre as mesmas, visto que a costa brasileira é muito ampla e as tendências climáticas têm variado bastante ao longo das últimas décadas.

\section{REFERÊNCIAS BIBLIOGRÁFICAS}

ANDRADE, A. F. de; COSTA, D. P. Pesquisa e desenvolvimento de boias meteoceanográficas. In: V Simpósio Brasileiro de Oceanografia, Santos. CD de Resumos, 2011.

BANCO DE DADOS OCEANOGRÁFICOS. Dados oceanográficos de temperatura, salinidade e superfície. Disponível em: http://www.goosbrasil.org/pirata/dados. Acessado em: 10 set. 2015.

BOURLĖS, B; LUMPKIN, R; MCPHADEN, M. J.; HERNANDEZ, F.; NOBRE, P.; CAMPOS E; YU, L.; PLANTON, S.; BUSALACCHI A.; MOURA, A. D.; SERVAIN, J.; TROTTE, J. The PIRATA program: History, accomplishments, and future directions. Bulletin of the American Meteorological Society, v. 89, n. 8, p. 1111-1126, 2008.

BOX, G. E. P.; JENKINS, G. M.; REINSEL, G. C.Time series analysis: forecasting and control. John Wiley \& Sons, 2015.

BROCKWELL, P.; RICHARD, D. Introduction to time series and forecasting.Springer, 2016.

CALADO, L. Dinâmica da interação da atividade de meso-escala da Corrente do Brasil com o fenômeno da ressurgência costeira ao largo de Cabo Frio e Cabo de São Tomé, RJ. Tese de Doutorado. Universidade de São Paulo. 2006.

CAPITÃ0, R.; FORTES, C. Análise comparativa entre estimativas do modelo SWAN e medições de agitação marítima efectuadas na Praia da Amoreira, Portugal. Revista de Gestão Costeira Integrada-Journal of Integrated Coastal Zone Management, v. 11, n. 3, p. 283-296, 2011.

CAVALCANTI, E. P.; SILVA, V. de P. R.; SOUSA, F. de A. S. Programa computacional para a estimativa da temperatura do ar para a região Nordeste do Brasil. Revista Brasileira de Engenharia Agrícola e Ambiental, v. 10, n. 1, p. 140-147, 2006.

CHECHI, L;; OLIVEIRA SANCHES, F de. Análise de uma série temporal de precipitação para Erechim (RS) e um possível método de previsão climática. Ambiência, v. 9, n. 1, p. 43-55, 2013.

CODATO, G. A. S.; WATANABE, W. B.; CALADO, L.; MARTINS, N.; RAMOS, A. E. A influência da frente térmica da ressurgência costeira de cabo frio na perda do sinal acústico: um estudo numérico. $X$ Encontro de Tecnologia Acústica Submarina-ETAS, 2011.

CORDEIR0, N. G. F. Estudo do afloramento ibérico: filamentos e climatologia superficial. Dissertação (Mestrado). Universidade de Aveiro. Departamento de Física. 2009.

CORDER, G. W.; F0REMAN, D. I. Nonparametric statistics: A step-by-step approach. John Wiley \& Sons, 2014.

DOUKHAN, P. Stochastic models for time series. Berlin: Springer, 2018.

ECHEVERRY, G. E. S.; TOLOI, C. M. de C. Testes para comparação de séries temporais: uma aplicação a séries de temperatura e salinidade da água, medidas em profundidades diferentes. Rev. Bras. Estat, p. 51-80, 2000.

GLOBAL SEA LEVEL OBSERVING SYSTEM. Informações sobre sistemas de monitoramento oceanográfico. Disponível em: https://www.gloss-sealevel.org. Acesso em: 20 out. 2015.

JAMES, G.; WITTEN, D; HASTIE, T.; TIBSHIRANI, R. An introduction to statistical learning. New York: Springer, 2013.

JONATHAN, D. C.; KUNG-SIK, C. Time series analysis with applications in R. SpringerLink, Springer eBooks, 2008. 
LAURINDO, L.; POLITO, P. Cálculo de perfis sintéticos de temperatura para os oceanos entre 39,5S e 39,5N a partir de dados de satélite, climatológicos e in situ. In: XIV Congresso Latino-Americano de Ciências do Mar, Balneário Camboriú, SC., 2015.

LEWIS, C. D. Demand Forecasting and Inventory Control.New York: Wiley, 157p 1997.

MARINHA DO BRASIL, Navio de Pesquisa Hidroceanográfico "Vital de Oliveira" realiza rodízio e manutenção de boias meteoceanográficas. Disponível em: https://www.marinha.mil.br/noticias/navio-de-pesquisa-hidroceanograficovital-de-oliveira-realiza-rodizio-e-manutenca0-de-boias. Acesso em: 14 mai. 2019.

MENDONCA, L. F.; SOUZA, R. B. de; REIS, R. N.; ALVES, R. de C. M. Análise da Variabilidade Superficial de Temperatura e Altimetria no 0ceano Atlântico Sudoeste durante o Ano de 2012. Revista Brasileira de Cartografia, v. 70, n. 3, p. $1158-1176,2018$.

MESQUITA, J. L. Mar Sem Fim. Disponível em: https://www.marinha.mil.br/noticias/navio-de-pesquisahidroceanografico-vital-de-oliveira-realiza-rodizio-e-manutencao-de-boias. Acesso em: 14 jan. 2021. 2017.

MOLLERI, G. S.; NOVO, E. M. de M.; KAMPEL, M. Space-time variability of the Amazon River plume based on satellite ocean color. Continental Shelf Research, v. 30, n. 3-4, p. 342-352, 2010.

MORETTIN, P. A.; TOLOI, C. M. de C. Análise de séries temporais. In: Análise de séries temporais. ABE, Blucher, 2006.

OLIVEIRA, A. C. S. de; SOUZA, A. A. de; LACERDA, W. S.; GONCALVES, L. R. Aplicação de redes neurais artificiais na previsão da produção de álcool. Ciênc. Agrotec. [online], vol.34, n.2, pp.279-284. ISSN 1413-7054. 2010.

PAULA, N. F. de.; SOUZA, R. B. de. Análise estatística de séries temporais de dados provenientes de sensores remotos e reanálises na região do oceano atlântico sudoeste. Ministério da Ciência e Tecnologia. Instituto Nacional de Pesquisas Espaciais, INPE. Santa Maria, 2010.

R CORE TEAM. R: A language and environment for statistical computing. R Foundation for Statistical Computing, Vienna, Austria. Disponível em: https://www.R-project.org/. 2019

SERVAIN, J.; CLAUZET, G.; WAINER, I. Times-scales of variability from the high frequency PIRATA data revealed by wavelet analysis. Revista Brasileira de Meteorologia, v. 20, n. 1, p. 43-58, 2005.

SIEGEL, S.; CASTELLAN JR., N. J. Nonparametric Statistics for the Behavioral Sciences. New York, 6. McGraw-Hill, 1988.

SILVA, G. L.; DOURADO, M. S.; CANDELLA, R. N. Estudo preliminar da climatologia da ressurgência da região de Arraial do Cabo, RJ. Anais do XI Encontro Nacional dos Grupos PET, UFSC, Florianópolis, SC, 1lp, 2006

TEIXEIRA, P. M. Uso de Técnicas de Mudança de Periodicidade de Séries Temporais para Previsão da Temperatura de Superfície do Mar do 0ceano Atlântico. Dissertação (Mestrado), Centro Federal de Educação Tecnológica Celso Suckow da Fonseca, CEFET/RJ, Rio de Janeiro. 2014.. 